\title{
Existence and Approximation of Solutions to Dynamic Inclusions in Time Scales
}

\section{Existência e Aproximação de Soluções para Inclusões Dinâmicas em Escalas de Tempo}

\author{
Iguer Luis Domini dos Santos \\ Faculdade de Engenharia do Campus de Ilha Solteira - Universidade Estadual Paulista \\ iguerluis@mat.feis.unesp.br
}

\begin{abstract}
Resumo: Este trabalho contribui para a existência de soluções para inclusões dinâmicas em escalas de tempo. Mais especificamente, prova um resultado de existência e aproximação de soluções para inclusões dinâmicas em escalas de tempo. O resultado obtido é uma generalização da existência e aproximação de soluções para inclusões diferenciais.
\end{abstract}

Palavras-chave: inclusões dinâmicas; existência de soluções; escalas de tempo.

\begin{abstract}
This work contributes to the existence of solutions for dynamic inclusions in time scales. More specifically, it proves a result of existence and approximation of solutions for dynamic inclusions in time scales. The result obtained is a generalization of the existence and approximation of solutions for differential inclusions.
\end{abstract}

Keywords: dynamic inclusions; existence of solutions; time scales.

\section{Introduction}

Recently, considerable attention has been given to the study of the existence of solutions for dynamic inclusions in time scales. This can be witnessed by works $[1,2,3,4,5,6,7]$. The work [1] proves the existence of solutions to first order dynamical inclusions in time scales with general boundary conditions. [2] investigates the existence of solutions and extrernal solutions for a first order impulsive dynamic inclusion on time scales. [3] proves the existence of solutions for second order dynamic inclusions in time scales with boundary conditions. The work [4] proves the existence of solutions for first order dynamic inclusions on time scales with nonlocal initial conditions. [5] studies existence results for systems of first order inclusions on time scales with an initial or a periodic boundary value condition. [6] studies the existence of solutions to nabla differential equations and nabla differential inclusions on time scales. [7] provides existence of solutions to a system of dynamical inclusions in time scales.

To the best of our knowledge, the approximation of solutions for dynamic inclusions in time scales has not been considered in the literature of time scales. In this work we have established a result of existence and approximation of solutions for dynamic inclusions in time scales.

Recebido em 01/04/2016 - Aceito em 07/07/2017

\begin{tabular}{lllll} 
RECEN & $19(1)$ & p.80-96 jan/jun & 2017 & DOI:10.5935/RECEN.2017.01.07 \\
\hline
\end{tabular}




\section{Background and preliminaries}

In this section, we gather basic concepts and results that will be useful in the development of the work.

We make the following conventions:

(i) if $x \in \mathbb{R}^{n}$ we denote the Euclidean norm of $x$ by $\|x\|$;

(ii) $B$ is the closed unit ball $\left\{x \in \mathbb{R}^{n}:\|x\| \leq 1\right\}$;

(iii) given a compact subset $E \subset \mathbb{R}$ and a function $g: E \rightarrow \mathbb{R}^{n}$, we will indicate by $\|g\|_{\infty}$ the supremum norm.

\section{$2.1 \quad$ Time scales}

A time scale is a nonempty closed subset $\mathbb{T} \subset \mathbb{R}$ of the real numbers. An arbitrary bounded time scale $\mathbb{T}$ will be taken, such that $a=\min \mathbb{T}$ and $b=\max \mathbb{T}$. We also suppose that $a<b$.

We define the forward jump operator $\sigma: \mathbb{T} \rightarrow \mathbb{T}$ by

$$
\sigma(t)=\inf \{s \in \mathbb{T}: s>t\}
$$

and the backward jump operator $\rho: \mathbb{T} \rightarrow \mathbb{T}$ by

$$
\rho(t)=\sup \{s \in \mathbb{T}: s<t\} .
$$

Here we assume that $\inf \emptyset=\sup \mathbb{T}$ and $\sup \emptyset=\inf \mathbb{T}$.

Lemma 1 (Cabada [8]). There exist $I \subset \mathbb{N}$ and $\left\{t_{i}\right\}_{i \in I} \subset \mathbb{T}$ such that

$$
R S:=\{t \in \mathbb{T}: t<\sigma(t)\}=\left\{t_{i}\right\}_{i \in I},
$$

where $R S$ stands for right scattered points of the time scale $\mathbb{T}$.

Define the function $\mu: \mathbb{T} \rightarrow[0,+\infty)$ by

$$
\mu(t)=\sigma(t)-t .
$$

If $A \subset \mathbb{R}$, we define the set $A_{\mathbb{T}}$ by $A_{\mathbb{T}}=A \cap \mathbb{T}$. We define $\mathbb{T}^{\kappa}:=\mathbb{T} \backslash(\rho(\sup \mathbb{T}) \text {, sup } \mathbb{T}]_{\mathbb{T}}$.

Consider a function $f: \mathbb{T} \rightarrow \mathbb{R}$ and $t \in \mathbb{T}^{\kappa}$. If $\xi \in \mathbb{R}$ is such that, for all $\varepsilon>0$ there exists $\delta>0$ obeying

$$
|f(\sigma(t))-f(s)-\xi(\sigma(t)-s)| \leq \varepsilon|\sigma(t)-s|
$$

for all $s \in(t-\delta, t+\delta)_{\mathbb{T}}$, we say that $\xi$ is the delta derivative of $f$ at $t$ and we denote it by $\xi:=f^{\Delta}(t)$.

Now, consider a function $f: \mathbb{T} \rightarrow \mathbb{R}^{n}$ and $t \in \mathbb{T}^{\kappa}$. We say that $f$ is $\Delta$-differentiable at $t$ if each component $f_{i}: \mathbb{T} \rightarrow \mathbb{R}$ of $f$ is $\Delta$-differentiable at $t$. In this case $f^{\Delta}(t)=$ $\left(f_{1}^{\Delta}(t), \ldots, f_{n}^{\Delta}(t)\right)$.

The next result is proven in [9] for scalar valued functions. But the generalization for vector valued functions is straightforward. 
Revista Ciências Exatas e Naturais, Vol.19, nº.1, Jan/Jun, 2017

Theorem 1 (Bohner [9]). Consider a function $f: \mathbb{T} \rightarrow \mathbb{R}^{n}$ and $t \in \mathbb{T}^{\kappa}$. Then the following statements hold:

(i) If $f$ is $\Delta$-differentiable at then $f$ is continuous at $t$.

(ii) If $f$ is continuous at $t$ and $\sigma(t)>t$, then $f$ is $\Delta$-differentiable at $t$. Furthermore,

$$
f^{\Delta}(t)=\frac{f(\sigma(t))-f(t)}{\mu(t)} .
$$

(iii) If $\sigma(t)=t$, then $f$ is $\Delta$-differentiable at $t$ if and only if there exists the limit

$$
\lim _{s \stackrel{\mathbb{T}}{\rightarrow} t} \frac{f(t)-f(s)}{t-s}
$$

as an element of $\mathbb{R}^{n}$. In that case

$$
f^{\Delta}(t)=\lim _{s \underset{\mathbb{T}}{\rightarrow} t} \frac{f(t)-f(s)}{t-s} .
$$

(iv) If $f$ is $\Delta$-differentiable at $t$, then

$$
f(\sigma(t))=f(t)+\mu(t) f^{\Delta}(t) .
$$

\section{$2.2 \Delta$-measurable sets}

Below, we recall the $\sigma$-algebra of subsets of the time scale $\mathbb{T}$.

Denote by $\mathfrak{F}$ the collection of all subintervals of $\mathbb{T}$ given by $[\tilde{a}, \tilde{b})_{\mathbb{T}}=\{t \in \mathbb{T}: \tilde{a} \leq t<\tilde{b}\}$, where $\tilde{a}, \tilde{b} \in \mathbb{T}$. The interval $[\tilde{a}, \tilde{a})_{\mathbb{T}}$ is understood as an empty set.

Take an arbitrary subset $E \subset \mathbb{T}$. If there exists at least one sequence of intervals $\left[a_{j}, b_{j}\right)_{\mathbb{T}} \in \mathfrak{F}$ such that $E \subset \bigcup_{j}\left[a_{j}, b_{j}\right)_{\mathbb{T}}$, the outer measure of $E$ is defined by

$$
m^{*}(E)=\inf \left\{\sum_{k=1}^{+\infty}\left(b_{k}-a_{k}\right): E \subset \bigcup_{k}\left[a_{k}, b_{k}\right)_{\mathbb{T}},\left[a_{k}, b_{k}\right)_{\mathbb{T}} \in \mathfrak{F}\right\} .
$$

It there is no such a cover of $E$ we set $m^{*}(E)=+\infty$.

The outer measure defined on $\mathbb{R}$ will be denoted by $\lambda^{*}$.

Properties of the outer measure $m^{*}$ can be founded in [8], [10], and [11]. Below, we have considered some of these properties.

Lemma 2 (Guseinov [10]). If $c, d \in \mathbb{T}$ and $c<d$ then

$$
m^{*}\left([c, d)_{\mathbb{T}}\right)=d-c .
$$

Using [11] one can prove the following lemma.

Lemma 3 (Royden [11]). If $\left\{E_{i}\right\}_{i \in \mathbb{N}}$ is a sequence of subsets of $\mathbb{T}$, then

$$
m^{*}\left(\bigcup_{i=1}^{+\infty} E_{i}\right) \leq \sum_{i=1}^{+\infty} m^{*}\left(E_{i}\right) .
$$


Lemma 4 (Cabada [8]). Let $E \subset[a, b)_{\mathbb{T}}$ be such that $E \subset\{t \in \mathbb{T}: \sigma(t)=t\}$. Then

$$
m^{*}(E)=\lambda^{*}(E) .
$$

Definition 1. A set $E \subset \mathbb{T}$ is said to be $\Delta$-measurable (Lebesgue $\Delta$-measurable) if

$$
m^{*}(A)=m^{*}(A \cap E)+m^{*}(A \cap(\mathbb{T} \backslash E))
$$

for each $A \subset \mathbb{T}$.

Proposition 1 (Cabada [8]). Take $E \subset \mathbb{T}$. Then $E$ is $\Delta$-measurable if and only if $E$ is Lebesgue measurable.

Thus we have the following result.

Theorem 2. The family of $\Delta$-measurable sets is a $\sigma$-algebra of $\mathbb{T}$.

We will indicate by $\Delta$ the $\sigma$-algebra of $\Delta$-measurable sets of $\mathbb{T}$. We call the measure $m^{*}: \Delta \rightarrow[0,+\infty]$ of $\Delta$-measure of Lebesgue and denoted it by $m^{*} \equiv \mu_{\Delta}$.

Let $E \subset \mathbb{T}$. We say that a statement $P$ holds $\Delta$-almost everywhere $(\Delta$-a.e. $)$ on $E$, if the set $N$ given by

$$
N=\{t \in E: P \text { does not hold at } t\}
$$

satisfies $\mu_{\Delta}(N)=0$.

\section{$2.3 \Delta$-measurable functions and $\Delta$-integrability}

We say that a function $f: \mathbb{T} \rightarrow[-\infty,+\infty]$ is $\Delta$-measurable if for each $r \in \mathbb{R}$ the set $\{t \in \mathbb{T}: f(t)<r\}$ is $\Delta$-measurable. A vector valued function $f: \mathbb{T} \rightarrow \mathbb{R}^{n}$ is said to be $\Delta$-measurable if each component $f_{i}: \mathbb{T} \rightarrow \mathbb{R}$ of $f$ is $\Delta$-measurable.

Given a function $f: \mathbb{T} \rightarrow \mathbb{R}^{n}$ define $\tilde{f}:[a, b] \rightarrow \mathbb{R}^{n}$ by

$$
\tilde{f}(t)=\left\{\begin{array}{l}
f(t), t \in \mathbb{T} \\
f\left(t_{i}\right), \quad t \in\left(t_{i}, \sigma\left(t_{i}\right)\right) \text { for some } i \in I
\end{array}\right.
$$

where $I \subset \mathbb{N}$ and $\left\{t_{i}\right\}_{i \in I} \subset \mathbb{T}$ are such that $\{t \in \mathbb{T}: t<\sigma(t)\}=\left\{t_{i}\right\}_{i \in I}$.

Proposition 2 (Cabada [8]). Consider a function $f: \mathbb{T} \rightarrow \mathbb{R}^{n}$. Then $f$ is $\Delta$-measurable if and only if $\tilde{f}$ is $\mathcal{L}$-measurable.

Proof. This result is stated in [8] for scalar valued functions $f$. However, it can be verified that it remains valid for vector valued functions.

For functions $f: \mathbb{T} \rightarrow \overline{\mathbb{R}}$ the integration concept can be found, for example, in [11] and [14]. The integral of a function $f: \mathbb{T} \rightarrow \overline{\mathbb{R}}$ over a set $E \in \Delta$ is denoted by

$$
\int_{E} f(s) \Delta s
$$

We call this integral the Lebesgue $\Delta$-integral of $f$ over $E$ and denote the set of functions $f: \mathbb{T} \rightarrow \mathbb{R}$ which are $\Delta$-integrable over $E$ by $L_{1}(E)$. If $f: \mathbb{T} \rightarrow \mathbb{R}^{n}$ is a $\Delta$-measurable function and $E \in \Delta, f$ is integrable over $E$ if each component $f_{i}: \mathbb{T} \rightarrow \mathbb{R}$ is integrable over $E$. Denote by $L_{1}\left(E, \mathbb{R}^{n}\right)$ the set of functions $f: \mathbb{T} \rightarrow \mathbb{R}^{n} \Delta$-integrable over $E$. 
Revista Ciências Exatas e Naturais, Vol.19, nº.1, Jan/Jun, 2017

Next, a result that relates the Lebesgue $\Delta$-integral in time scales and the usual Lebesgue integral is presented.

If $E \subset \mathbb{T}$ we define the set $\tilde{E}$ by

$$
\tilde{E}=E \cup \bigcup_{i \in I_{E}}\left(t_{i}, \sigma\left(t_{i}\right)\right)
$$

where

$$
I_{E}:=\left\{i \in I: t_{i} \in E \cap R S\right\} .
$$

The following result is provided in [8] for scalar valued functions. However, one can see that it holds for vector valued functions as stated below.

Theorem 3 (Cabada [8]). Take a $\Delta$-measurable set $E \subset \mathbb{T}$ such that $b \notin E$. Let $f: \mathbb{T} \rightarrow \mathbb{R}^{n}$ be a $\Delta$-measurable function and $\tilde{f}:[a, b] \rightarrow \mathbb{R}^{n}$ the extension of $f$ previously defined. Then $f \in L_{1}\left(E, \mathbb{R}^{n}\right)$ if and only if $\tilde{f} \in L_{1}\left(\tilde{E}, \mathbb{R}^{n}\right)$. In this case

$$
\int_{E} f(s) \Delta s=\int_{\tilde{E}} \tilde{f}(s) d s
$$

The vector space $L_{1}\left([a, b)_{\mathbb{T}}, \mathbb{R}^{n}\right)$ equipped with the norm

$$
\|f\|_{1}=\int_{[a, b)_{\mathbb{T}}}\|f(s)\| \Delta s
$$

is a Banach space, as stated below.

Theorem 4. $L_{1}\left([a, b)_{\mathbb{T}}, \mathbb{R}^{n}\right)$ is a Banach space.

We also have the following theorem.

Theorem 5. Let $\left\{f_{j}\right\}$ be a sequence in $L_{1}\left([a, b)_{\mathbb{T}}, \mathbb{R}^{n}\right)$ and $f \in L_{1}\left([a, b)_{\mathbb{T}}, \mathbb{R}^{n}\right)$ such that $\left\|f_{j}-f\right\|_{1} \rightarrow 0$. Then there exists a subsequence $\left\{f_{j_{m}}\right\}$ satisfying

(i) $f_{j_{m}}(t) \rightarrow f(t)$ for $\Delta$-a.e. $t \in[a, b)_{\mathbb{T}}$;

(ii) $\left\|f_{j_{m}}(t)\right\| \leq h(t) \forall m$ and for $\Delta$-a.e. $t \in[a, b)_{\mathbb{T}}$, where $h \in L_{1}\left([a, b)_{\mathbb{T}}, \mathbb{R}^{n}\right)$.

Theorems 4 and 5 are obtained similar to [[12], Théorème IV.8.] and [[12], Théorème IV.9.], respectively.

We will use the following elementary result in the proof of lemma 6 .

Lemma 5. Let $k:[a, b] \rightarrow \mathbb{R}$ be a function in $L_{1}([a, b])$. For each $j \geq 1$ and $t \in[a, b]$ we have

$$
\int_{a}^{t} k\left(s_{1}\right) \int_{a}^{s_{1}} k\left(s_{2}\right) \cdots \int_{a}^{s_{j-1}} k\left(s_{j}\right) d s_{j} \cdots d s_{2} d s_{1}=\frac{\left[\int_{a}^{t} k(\tau) d \tau\right]^{j}}{j !}
$$

where $s_{0}=t$.

Lemma 6. Consider a function $k: \mathbb{T} \rightarrow[0, \infty)$ in $L_{1}\left([a, b)_{\mathbb{T}}\right)$. If

$$
\eta(t)=\int_{[a, t)_{\mathbb{T}}} k\left(s_{1}\right) \int_{\left[a, s_{1}\right)_{\mathbb{T}}} k\left(s_{2}\right) \cdots \int_{\left[a, s_{j-1}\right)_{\mathbb{T}}} k\left(s_{j}\right) \Delta s_{j} \cdots \Delta s_{2} \Delta s_{1}
$$


SANTOS, I. L. D. dos

we have

$$
\eta(t) \leq \frac{\left[\int_{[a, t)_{\mathbb{T}}} k(\tau) \Delta \tau\right]^{j}}{j !}
$$

for all $j \geq 1$ and all $t \in \mathbb{T}$, where $s_{0}=t$.

Proof. Define $g: \mathbb{T} \rightarrow[0, \infty)$ by $g(s)=\int_{[a, s)_{\mathbb{T}}} k(\tau) \Delta \tau$. Then

$$
\begin{aligned}
\int_{[a, t)_{\mathbb{T}}} k\left(s_{1}\right) \int_{\left[a, s_{1}\right)_{\mathbb{T}}} k\left(s_{2}\right) \Delta s_{2} \Delta s_{1} & =\int_{[a, t)_{\mathbb{T}}} k\left(s_{1}\right) g\left(s_{1}\right) \Delta s_{1} \\
& =\int_{[a, t)} \tilde{k}\left(s_{1}\right) \tilde{g}\left(s_{1}\right) d s_{1} .
\end{aligned}
$$

If $s_{1} \in \mathbb{T}$ it follows that

$$
\tilde{g}\left(s_{1}\right)=g\left(s_{1}\right)=\int_{\left[a, s_{1}\right)_{\mathbb{T}}} k\left(s_{2}\right) \Delta s_{2}=\int_{\left[a, s_{1}\right)} \tilde{k}\left(s_{2}\right) d s_{2} .
$$

However, if $s_{1} \notin \mathbb{T}$ let $i \in I$ be such that $s_{1} \in\left(t_{i}, \sigma\left(t_{i}\right)\right)$. We have

$$
\begin{aligned}
\tilde{g}\left(s_{1}\right) & =g\left(t_{i}\right)=\int_{\left[a, t_{i}\right)_{\mathbb{T}}} k\left(s_{2}\right) \Delta s_{2} \\
& =\int_{\left[a, t_{i}\right)} \tilde{k}\left(s_{2}\right) d s_{2} \leq \int_{\left[a, s_{1}\right)} \tilde{k}\left(s_{2}\right) d s_{2}
\end{aligned}
$$

and then

$$
\begin{aligned}
\int_{[a, t)_{\mathbb{T}}} k\left(s_{1}\right) g\left(s_{1}\right) \Delta s_{1} & \leq \int_{[a, t)} \tilde{k}\left(s_{1}\right) \int_{\left[a, s_{1}\right)} \tilde{k}\left(s_{2}\right) d s_{2} d s_{1} \\
& =\frac{\left[\int_{[a, t)} \tilde{k}(\tau) d \tau\right]^{2}}{2 !}=\frac{\left[\int_{[a, t)_{\mathbb{T}}} k(\tau) \Delta \tau\right]^{2}}{2 !} .
\end{aligned}
$$

Suppose the lemma is valid for $j \geq 2$. Below we find that the lemma is also valid for $j+1$ and therefore by mathematical induction we conclude that the formula 1 is valid for all $j$.

Let $g: \mathbb{T} \rightarrow[0, \infty)$ be defined by

$$
g\left(s_{1}\right)=\int_{\left[a, s_{1}\right)_{\mathbb{T}}} k\left(s_{2}\right) \cdots \int_{\left[a, s_{j}\right)_{\mathbb{T}}} k\left(s_{j+1}\right) \Delta s_{j+1} \cdots \Delta s_{2} .
$$

If $s_{1} \in \mathbb{T}$ we have

$$
\tilde{g}\left(s_{1}\right)=g\left(s_{1}\right) \leq \frac{\left[\int_{\left[a, s_{1}\right)_{\mathbb{T}}} k(\tau) \Delta \tau\right]^{j}}{j !}=\frac{\left[\int_{\left[a, s_{1}\right)} \tilde{k}(\tau) d \tau\right]^{j}}{j !}
$$

and if $s_{1} \notin \mathbb{T}$ let $i \in I$ be such that $s_{1} \in\left(t_{i}, \sigma\left(t_{i}\right)\right)$, thence

$$
\begin{aligned}
\tilde{g}\left(s_{1}\right) & =g\left(t_{i}\right) \leq \frac{\left[\int_{\left[a, t_{i}\right)_{\mathbb{T}}} k(\tau) \Delta \tau\right]^{j}}{j !} \\
& =\frac{\left[\int_{\left[a, t_{i}\right)} \tilde{k}(\tau) d \tau\right]^{j}}{j !} \leq \frac{\left[\int_{\left[a, s_{1}\right)} \tilde{k}(\tau) d \tau\right]^{j}}{j !}
\end{aligned}
$$


Revista Ciências Exatas e Naturais, Vol.19, nº.1, Jan/Jun, 2017

and thus

$$
\begin{aligned}
\int_{[a, t)_{\mathbb{T}}} k\left(s_{1}\right) g\left(s_{1}\right) \Delta s_{1} & =\int_{[a, t)} \tilde{k}\left(s_{1}\right) \tilde{g}\left(s_{1}\right) d s_{1} \\
& \leq \int_{[a, t)} \tilde{k}\left(s_{1}\right) \frac{\left[\int_{\left[a, s_{1}\right)} \tilde{k}(\tau) d \tau\right]^{j}}{j !} d s_{1} \\
& =\frac{\left[\int_{[a, t)} \tilde{k}(\tau) d \tau\right]^{j+1}}{(j+1) !}=\frac{\left[\int_{[a, t)_{\mathbb{T}}} k(\tau) \Delta \tau\right]^{j+1}}{(j+1) !} .
\end{aligned}
$$

\subsection{Absolutely continuous functions in time scales}

A function $f: \mathbb{T} \rightarrow \mathbb{R}^{n}$ is absolutely continuous if given $\varepsilon>0$ there exists $\delta>0$ such that

$$
\sum_{i=1}^{N}\left\|f\left(b_{i}\right)-f\left(a_{i}\right)\right\|<\varepsilon
$$

whenever $a_{i} \leq b_{i}$ and $\left\{\left[a_{i}, b_{i}\right)_{\mathbb{T}}\right\}_{i=1}^{N}$ are disjoint intervals obeying

$$
\sum_{i=1}^{N}\left(b_{i}-a_{i}\right)<\delta
$$

Theorem 6 given below is established in [13] for scalar valued functions. However, it is easy to see that it can be extended for vector valued functions as stated below.

Theorem 6 (Cabada [13]). A function $f: \mathbb{T} \rightarrow \mathbb{R}^{n}$ is absolutely continuous if and only if the following assertions are valid:

(i) $f$ is $\Delta$-differentiable $\Delta$-a.e. on $[a, b)_{\mathbb{T}}$ and $f^{\Delta} \in L_{1}\left([a, b)_{\mathbb{T}}, \mathbb{R}^{n}\right)$;

(ii) for each $t \in \mathbb{T}$ we have

$$
f(t)=f(a)+\int_{[a, t)_{\mathbb{T}}} f^{\Delta}(s) \Delta s .
$$

We say that the function $f: \mathbb{T} \rightarrow \mathbb{R}^{n}$ is an arc if it is absolutely continuous.

In the next lemma, we get a result on absolutely continuous functions in time scales.

Proposition 3 (Santos [7]). Let $f: \mathbb{T} \rightarrow[0,+\infty)$ be a function in $L_{1}\left([a, b)_{\mathbb{T}}\right)$. Given $\varepsilon>0$ there exists $\delta>0$ such that, if $A \in \Delta$ and $\mu_{\Delta}(A)<\delta$ then

$$
\int_{A} f(s) \Delta s<\varepsilon
$$

Lemma 7. If $v \in L_{1}\left([a, b)_{\mathbb{T}}, \mathbb{R}^{n}\right)$ and $x_{0} \in \mathbb{R}^{n}$, then the function $z: \mathbb{T} \rightarrow \mathbb{R}^{n}$ given by

$$
z(t)=x_{0}+\int_{[a, t)_{\mathbb{T}}} v(s) \Delta s
$$

is an arc. Furthermore

$$
z^{\Delta}(t)=v(t) \quad \Delta-\text { a.e. } t \in[a, b)_{\mathbb{T}} .
$$


Proof. The function $z$ is an arc because of Proposition 3 .

Let $t \in[a, b)_{\mathbb{T}}$ be such that there exists the delta derivative $z^{\Delta}(t)$. If $\sigma(t)>t$ we have

$$
\begin{aligned}
z^{\Delta}(t) & =\frac{\int_{[a, \sigma(t))_{\mathbb{T}}} v(s) \Delta s-\int_{[a, t)_{\mathbb{T}}} v(s) \Delta s}{\mu(t)} \\
& =\frac{\int_{[t, \sigma(t))_{\mathbb{T}}} v(s) \Delta s}{\mu(t)}=v(t) .
\end{aligned}
$$

If $\sigma(t)=t$ and $t$ is a Lebesgue point of $\tilde{v}$, consider a sequence $\left\{t_{j}\right\}_{j \in \mathbb{N}} \subset[a, b)_{\mathbb{T}}$ such that $t_{j} \downarrow t$. It follows from Theorem 3 and property Lebesgue point [14] that

$$
\begin{aligned}
z^{\Delta}(t) & =\lim _{j \rightarrow \infty} \frac{z\left(t_{j}\right)-z(t)}{t_{j}-t}=\lim _{j \rightarrow \infty} \frac{\int_{\left[t, t_{j}\right)_{\mathbb{T}}} v(s) \Delta s}{t_{j}-t} \\
& =\lim _{j \rightarrow \infty} \frac{\int_{\left[t, t_{j}\right)} \tilde{v}(s) d s}{t_{j}-t}=\tilde{v}(t)=v(t) .
\end{aligned}
$$

Thence, if $D=\left\{t \in[a, b)_{\mathbb{T}}: z^{\Delta}(t) \neq v(t)\right\}$ we have $D \subset A \cap E$, where

$$
A=\left\{t \in[a, b)_{\mathbb{T}}: \sigma(t)=t\right\}
$$

and $E$ is the set of points $t \in[a, b)_{\mathbb{T}}$ such that $t$ is not a Lebesgue point of $\tilde{v}$. Using Lemma 4 we conclude that

$$
m^{*}(D) \leq m^{*}(A \cap E)=\lambda^{*}(A \cap E) \leq \lambda^{*}(E)=0
$$

and thus $z^{\Delta}(t)=v(t) \Delta$-a.e. $t \in[a, b)_{\mathbb{T}}$.

\subsection{Set-valued functions properties}

Here we gather results of measurable multifunction that will be needed in the sequel.

Let $(\Omega, \mathcal{F})$ be a measurable space. A set-valued function $E: \Omega \rightsquigarrow \mathbb{R}^{n}$ is said to be $\mathcal{F}$-measurable if the set

$$
E^{-1}(V)=\{x \in \Omega: E(x) \cap V \neq \emptyset\}
$$

is $\mathcal{F}$-measurable for all compact sets $V \subset \mathbb{R}^{n}$.

A set-valued function $E$ is said to be closed or nonempty when its image $E(x)$ satisfies the required property, for each point $x \in \Omega$.

Lemma 8 (Castaing [15]). Take a measurable space $(\Omega, \mathcal{F})$ and a nonempty closed set-valued function $E: \Omega \rightsquigarrow \mathbb{R}^{n}$. If $E$ is $\mathcal{F}$-measurable, then $E$ has a measurable selection.

Lemma 9 (Castaing [15]). Let $E:[a, b] \rightsquigarrow \mathbb{R}^{m}$ be a closed set-valued function and $\Gamma:=$ $\{t \in[a, b]: E(t) \neq \emptyset\}$. Then the following statements are equivalent:

(i) The set-valued function $E$ is $\mathcal{L}$-measurable;

(ii) The set $\operatorname{Gr} E=\{(t, v): v \in E(t)\}$ is $\mathcal{L} \times \mathcal{B}^{m}$-measurable;

(iii) The set $\Gamma$ is $\mathcal{L}$-measurable and there is a sequence of $\mathcal{L}$-measurable functions $\gamma_{i}: \Gamma \rightarrow$ $\mathbb{R}^{m}$ such that

$$
E(t)=\overline{\left\{\gamma_{i}(t): i=1,2, \ldots\right\}} \text { for each } t \in \Gamma .
$$


Revista Ciências Exatas e Naturais, Vol.19, nº.1, Jan/Jun, 2017

We have the following consequence of the previous lemma.

Corollary 1. Let $E_{1}, E_{2}:[a, b] \rightsquigarrow \mathbb{R}^{m}$ be closed set-valued functions. If $E_{1}$ and $E_{2}$ are $\mathcal{L}$-measurable, then the set-valued function $E:[a, b] \rightsquigarrow \mathbb{R}^{m}$ given by

$$
E(t)=E_{1}(t) \cap E_{2}(t)
$$

is $\mathcal{L}$-measurable.

Consider a function $\phi: \mathbb{T} \times \mathbb{R}^{m} \rightarrow \mathbb{R}^{n}$. We say that $\phi$ is a $\Delta$-Carathéodory function if it satisfies the followings properties:

(i) for each $t \in \mathbb{T}$, the function $x \mapsto \phi(t, x)$ is continuous.

(ii) for each $x \in \mathbb{R}^{m}$, the function $t \mapsto \phi(t, x)$ is $\Delta$-measurable.

Let $\mathcal{B}^{m}$ denote the Borel $\sigma$-algebra of $\mathbb{R}^{m}$. We use the notation $\Delta \times \mathcal{B}^{m}$ for the product $\sigma$-algebra between $\Delta$ and $\mathcal{B}^{m}$. We recall that the $\sigma$-algebra $\Delta \times \mathcal{B}^{m}$ is the least $\sigma$-algebra of $\mathbb{T} \times \mathbb{R}^{m}$ that contains all products $A \times B$, where $A \in \Delta$ and $B \in \mathcal{B}^{m}$.

Lemma 10 (Loewen $[16]$ ). Let $\phi:[a, b] \times \mathbb{R}^{m} \rightarrow \mathbb{R}^{n}$ be a $\mathcal{L}$-Carathéodory function. Then $\phi$ is $\mathcal{L} \times \mathcal{B}^{m}$-measurable.

Take a function $\phi: \mathbb{T} \times \mathbb{R}^{m} \rightarrow \mathbb{R}^{n}$. We define the function $\tilde{\phi}:[a, b] \times \mathbb{R}^{m} \rightarrow \mathbb{R}^{n}$ by

$$
\tilde{\phi}(t, u)=\left\{\begin{array}{l}
\phi(t, u), t \in \mathbb{T} \\
\phi\left(t_{i}, u\right), \quad t \in\left(t_{i}, \sigma\left(t_{i}\right)\right) \text { for some } i \in I .
\end{array}\right.
$$

Lemma 11 (Santos [17]). Let $\phi: \mathbb{T} \times \mathbb{R}^{m} \rightarrow \mathbb{R}^{n}$ be a $\Delta$-Carathéodory function. Then $\tilde{\phi}$ is a $\mathcal{L}$-Carathéodory function.

Lemma 12 (Santos [17]). Let $u: \mathbb{T} \rightarrow \mathbb{R}^{m}$ be a $\Delta$-measurable function. If $B \in \mathcal{B}^{m}$ then $u^{1}(B) \in \Delta$.

Proposition 4. Let $F: \mathbb{T} \times \mathbb{R}^{m} \rightsquigarrow \mathbb{R}^{n}$ be a $\Delta \times \mathcal{B}^{m}$-measurable set-valued function and $u: \mathbb{T} \rightarrow \mathbb{R}^{m}$ a $\Delta$-measurable function. Then the set-valued function $G: \mathbb{T} \rightsquigarrow \mathbb{R}^{n}$ defined by

$$
G(t)=F(t, u(t))
$$

is $\Delta$-measurable.

Proof. Denote by $\mathcal{D}$ the collection of subsets

$$
\mathcal{D}=\left\{E \subset \mathbb{T} \times \mathbb{R}^{m}:\{t \in \mathbb{T}:(t, u(t)) \in E\} \in \Delta\right\} .
$$

Then $\mathcal{D}$ is a $\sigma$-algebra in $\mathbb{T} \times \mathbb{R}^{m}$. If $A \in \Delta$ and $B \in \mathcal{B}^{m}$ it follows that

$$
\{t \in \mathbb{T}:(t, u(t)) \in A \times B\}=A \cap u^{-1}(B) \in \Delta .
$$

Thus $\Delta \times \mathcal{B}^{m} \subset \mathcal{D}$.

Take arbitrarily a compact set $V \subset \mathbb{R}^{n}$. We have

$$
E=\left\{(t, u) \in \mathbb{T} \times \mathbb{R}^{m}: F(t, u) \cap V \neq \emptyset\right\}=F^{-1}(V) \in \Delta \times \mathcal{B}^{m}
$$

and then $E \in \mathcal{D}$. Therefore

$$
G^{-1}(V)=\{t \in \mathbb{T}: F(t, u(t)) \cap V \neq \emptyset\}=\{t \in \mathbb{T}:(t, u(t)) \in E\} \in \Delta
$$

and $G$ is $\Delta$-measurable. 
Proposition 5. Take a $\Delta$-Carathéodory function $\phi: \mathbb{T} \times \mathbb{R}^{n} \rightarrow \mathbb{R}$ and a $\Delta$-measurable nonempty closed set-valued function $H: \mathbb{T} \rightsquigarrow \mathbb{R}^{n}$. Suppose that for each $t \in \mathbb{T}$ there exists $u \in H(t)$ such that $\phi(t, u)=0$. Thence the set-valued function $G: \mathbb{T} \rightsquigarrow \mathbb{R}^{n}$ given by

$$
G(t)=\{u \in H(t): \phi(t, u)=0\}
$$

has a $\Delta$-measurable selection.

Proof. Since $\phi$ is a $\Delta$-Carathéodory function, then $\tilde{\phi}:[a, b] \times \mathbb{R}^{n} \rightarrow \mathbb{R}$ is a $\mathcal{L}$-Carathéodory function. Hence $\tilde{\phi}$ is a $\mathcal{L} \times \mathcal{B}^{n}$-measurable function. have

If the set-valued function $\Phi:[a, b] \rightsquigarrow \mathbb{R}^{n}$ is defined as $\Phi(t)=\left\{u \in \mathbb{R}^{n}: \tilde{\phi}(t, u)=0\right\}$, we

$$
\begin{aligned}
G r \Phi & =\left\{(t, \gamma) \in[a, b] \times \mathbb{R}^{n}: \gamma \in \Phi(t)\right\} \\
& =\left\{(t, \gamma) \in[a, b] \times \mathbb{R}^{n}: \tilde{\phi}(t, \gamma)=0\right\} \\
& =\tilde{\phi}^{-1}(\{0\}) \in \mathcal{L} \times \mathcal{B}^{n}
\end{aligned}
$$

and it follows from Lemma 9 that $\Phi$ is $\mathcal{L}$-measurable, since $\Phi$ is closed.

Define $N:[a, b] \rightsquigarrow \mathbb{R}^{n}$ by $N(t)=\tilde{H}(t) \cap \Phi(t)$. As $\tilde{H}$ is a closed and $\mathcal{L}$-measurable set-valued function we deduce that $N$ is $\mathcal{L}$-measurable. Thence, for any compact subset $V \subset \mathbb{R}^{n}$ we have

$$
\begin{aligned}
G^{-1}(V) & =\{t \in \mathbb{T}: G(t) \cap V \neq \emptyset\} \\
& =\{t \in[a, b]: N(t) \cap V \neq \emptyset\} \cap \mathbb{T} \in \mathcal{L}
\end{aligned}
$$

that is, $G^{-1}(V) \in \Delta$ and then $G$ is $\Delta$-measurable.

Since $G$ is a nonempty closed and $\Delta$-measurable set-valued function, from Lemma 8 we see that $G$ has a $\Delta$-measurable selection.

Consider a set-valued function $F: \mathbb{T} \times \mathbb{R}^{n} \rightsquigarrow \mathbb{R}^{n}$. We say that $F$ satisfies the hypotheses (H1) and (H2) if

(H1) $F$ is a nonempty, closed and $\Delta \times \mathcal{B}^{n}$-measurable set-valued function.

(H2) there exists a function $k: \mathbb{T} \rightarrow[0, \infty)$ in $L_{1}\left([a, b)_{\mathbb{T}}\right)$ such that for each $t \in \mathbb{T}$,

$$
F(t, x) \subset F(t, y)+k(t)\|y-x\| B
$$

for all $x, y$ in $\mathbb{R}^{n}$.

To the set-valued function $F$ we associate the function $\rho: \mathbb{T} \times \mathbb{R}^{n} \times \mathbb{R}^{n} \rightarrow[0, \infty)$ given by

$$
\rho(t, x, v):=\inf \{|v-y|: y \in F(t, x)\} .
$$

Lemma 13. Let $F: \mathbb{T} \times \mathbb{R}^{n} \rightsquigarrow \mathbb{R}^{n}$ be a set-valued function satisfying the hypotheses (H1) and (H2). Then

(i) the function $t \mapsto \rho(t, x, v)$ is $\Delta$-measurable for each $(x, v)$ in $\mathbb{R}^{n} \times \mathbb{R}^{n}$.

(ii) for any $t$ in $\mathbb{T}$, and any $x_{1}, x_{2}, v_{1}, v_{2}$ in $\mathbb{R}^{n}$, one has

$$
\left|\rho\left(t, x_{1}, v_{1}\right)-\rho\left(t, x_{2}, v_{2}\right)\right| \leq k(t)\left\|x_{1}-x_{2}\right\|+\left\|v_{1}-v_{2}\right\| .
$$


Revista Ciências Exatas e Naturais, Vol.19, nº.1, Jan/Jun, 2017

Proof. (i) Take $\alpha \in \mathbb{R}$ arbitrary. If $\alpha<0$ we have

$$
\{t \in \mathbb{T}: \rho(t, x, v) \leq \alpha\}=\emptyset \in \Delta .
$$

On the other hand, if $\alpha \geq 0$ then

$$
\{t \in \mathbb{T}: \rho(t, x, v) \leq \alpha\}=\{t \in \mathbb{T}: F(t, x) \cap\{v+\alpha B\} \neq \emptyset\} \in \Delta
$$

since the set-valued function $t \rightsquigarrow F(t, x)$ is $\Delta$-measurable by Proposition 4 .

(ii) Fix an arbitrary $\delta>0$. So there exists $\eta_{2} \in F\left(t, x_{2}\right)$ such that

$$
\rho\left(t, x_{2}, v_{2}\right)>\left|v_{2}-\eta_{2}\right|-\delta .
$$

By the hypothesis (H2) there exists $\eta_{1} \in F\left(t, x_{1}\right)$ such that

$$
\left\|\eta_{1}-\eta_{2}\right\| \leq k(t)\left\|x_{1}-x_{2}\right\|
$$

As $\rho\left(t, x_{1}, v_{1}\right) \leq\left\|v_{1}-\eta_{1}\right\|$ it follows that

$$
\begin{aligned}
\rho\left(t, x_{1}, v_{1}\right)-\rho\left(t, x_{2}, v_{2}\right) & \leq\left\|v_{1}-\eta_{1}\right\|-\rho\left(t, x_{2}, v_{2}\right) \\
& <\left\|v_{1}-\eta_{1}\right\|-\left\|v_{2}-\eta_{2}\right\|+\delta \\
& \leq\left\|v_{1}-v_{2}\right\|+\left\|v_{2}-\eta_{1}\right\|-\left\|v_{2}-\eta_{2}\right\|+\delta \\
& \leq\left\|v_{1}-v_{2}\right\|+\left\|\eta_{2}-\eta_{1}\right\|+\delta \\
& \leq\left\|v_{1}-v_{2}\right\|+k(t)\left\|x_{1}-x_{2}\right\|+\delta .
\end{aligned}
$$

Similarly we obtain

$$
\rho\left(t, x_{2}, v_{2}\right)-\rho\left(t, x_{1}, v_{1}\right)<\left\|v_{1}-v_{2}\right\|+k(t)\left\|x_{1}-x_{2}\right\|+\delta .
$$

Since $\delta$ is arbitrary, the proof is complete.

Corollary 2. Let $F: \mathbb{T} \times \mathbb{R}^{n} \rightsquigarrow \mathbb{R}^{n}$ be a set-valued function obeying the hypotheses (H1) and (H2). If $x: \mathbb{T} \rightarrow \mathbb{R}^{n}$ is an arc, then the set-valued function

$$
t \rightsquigarrow\left\{u \in F(t, x(t)):\left\|u-x^{\Delta}(t)\right\|-\rho\left(t, x(t), x^{\Delta}(t)\right)=0\right\}
$$

has a $\Delta$-measurable selection.

Proof. First, note that the function

$$
t \mapsto \rho\left(t, x(t), x^{\Delta}(t)\right)
$$

is $\Delta$-measurable. Indeed, if $w: \mathbb{T} \rightarrow \mathbb{R}^{n} \times \mathbb{R}^{n}$ is given by $w(t)=\left(x(t), x^{\Delta}(t)\right)$, so $\tilde{w}:[a, b] \rightarrow$ $\mathbb{R}^{n} \times \mathbb{R}^{n}$ is $\mathcal{L}$-measurable. Since $\rho: \mathbb{T} \times \mathbb{R}^{2 n} \rightarrow \mathbb{R}$ is a $\Delta$-Carathéodory function, it follows that $\tilde{\rho}:[a, b] \times \mathbb{R}^{2 n} \rightarrow \mathbb{R}$ is a $\mathcal{L}$-Carathéodory function. It follows from Lemma 10 that the function $\tilde{\rho}$ is $\mathcal{L} \times \mathcal{B}^{2 n}$-measurable. Hence, the function

$$
t \mapsto \tilde{\rho}(t, \tilde{w}(t))
$$

is $\mathcal{L}$-measurable.

If $\alpha \in \mathbb{R}$ is arbitrarily fixed, we have

$$
\{t \in \mathbb{T}: \rho(t, w(t)<\alpha\}=\{t \in[a, b]: \tilde{\rho}(t, \tilde{w}(t))<\alpha\} \cap \mathbb{T}
$$

and so the function $t \mapsto \rho(t, w(t))$ is $\Delta$-measurable.

Since the function

$$
\phi(t, u)=\left\|u-x^{\Delta}(t)\right\|-\rho\left(t, x(t), x^{\Delta}(t)\right)
$$

is a $\Delta$-Carathéodory function, we conclude the proof. 
SANTOS, I. L. D. dos

\section{Existence and approximation of solutions}

Finally, in this section we obtain the main result.

In the next theorem, we suppose that the set-valued function $F: \mathbb{T} \times \mathbb{R}^{n} \rightsquigarrow \mathbb{R}^{n}$ obey the hypotheses (H1) and (H2), and we set

$$
K=\exp \left\{\int_{[a, b)_{\mathbb{T}}} k(t) \Delta t\right\} .
$$

If $x: \mathbb{T} \rightarrow \mathbb{R}^{n}$ is an arc, we define

$$
\rho_{F}(x)=\int_{[a, b)_{\mathbb{T}}} \rho\left(t, x(t), x^{\Delta}(t)\right) \Delta t .
$$

We also say that the $\operatorname{arc} x$ is a trajectory of $F$ if it satisfies the inclusion

$$
x^{\Delta}(t) \in F(t, x(t)) \quad \Delta-\text { a.e. } t \in[a, b)_{\mathbb{T}} .
$$

Theorem 7 (existence and approximation). Consider a set-valued function $F: \mathbb{T} \times \mathbb{R}^{n} \rightsquigarrow$ $\mathbb{R}^{n}$. Then for any arc $x$ and constant $\varepsilon>0$ satisfying $\rho_{F}(x)<\frac{\varepsilon}{K}$, there exists a trajectory $y$ of $F$ such that $y(a)=x(a)$ and

$$
\|x-y\|_{\infty} \leq \int_{[a, b)_{\mathbb{T}}}\left\|x^{\Delta}(s)-y^{\Delta}(s)\right\| \Delta s \leq K \rho_{F}(x) .
$$

Proof. The proof consists of the construction of a sequence of approximate trajectories $\left\{x_{j}\right\}$ starting from $x_{0} \equiv x$ by choosing $x_{j+1}^{\Delta}(t)$ as the nearest point of $F\left(t, x_{j}(t)\right)$ to $x_{j}^{\Delta}(t)$. We show that the sequence $\left\{x_{j}\right\}$ converges uniformly to the trajectory $y$ of the theorem.

Let $G_{0}: \mathbb{T} \rightsquigarrow \mathbb{R}^{n}$ be a set-valued function defined as

$$
G_{0}(t)=\left\{v \in F(t, x(t)):\left\|v-x^{\Delta}(t)\right\|-\rho\left(t, x(t), x^{\Delta}(t)\right)=0\right\} .
$$

Hence $G_{0}$ has a $\Delta$-measurable selection $v_{0}: \mathbb{T} \rightarrow \mathbb{R}^{n}$.

Since

$$
\left\|v_{0}(t)\right\| \leq\left\|x^{\Delta}(t)\right\|+\rho\left(t, x(t), x^{\Delta}(t)\right)
$$

we have $v_{0} \in L_{1}\left([a, b)_{\mathbb{T}}, \mathbb{R}^{n}\right)$. Thence the function $x_{1}: \mathbb{T} \rightarrow \mathbb{R}^{n}$ given by

$$
x_{1}(t)=x_{0}+\int_{[a, t)_{\mathbb{T}}} v_{0}(\tau) \Delta \tau
$$

is an arc and satisfies

$$
\begin{aligned}
\left\|x_{1}(t)-x(t)\right\| & =\left\|\int_{[a, t)_{\mathbb{T}}}\left(v_{0}(s)-x^{\Delta}(s)\right) \Delta s\right\| \\
& \leq \int_{[a, t)_{\mathbb{T}}}\left\|v_{0}(s)-x^{\Delta}(s)\right\| \Delta s \leq \int_{[a, b)_{\mathbb{T}}}\left\|v_{0}(s)-x^{\Delta}(s)\right\| \Delta s \\
& =\int_{[a, b)_{\mathbb{T}}} \rho\left(s, x(s), x^{\Delta}(s)\right) \Delta s=\rho_{F}(x)
\end{aligned}
$$

for all $t \in \mathbb{T}$. 
Revista Ciências Exatas e Naturais, Vol.19, nº.1, Jan/Jun, 2017

If the set-valued function $G_{1}: \mathbb{T} \rightsquigarrow \mathbb{R}^{n}$ is defined by

$$
G_{1}(t)=\left\{v \in F\left(t, x_{1}(t)\right):\left\|v-x_{1}^{\Delta}(t)\right\|-\rho\left(t, x_{1}(t), x_{1}^{\Delta}(t)\right)=0\right\}
$$

then $G_{1}$ has a $\Delta$-measurable selection $v_{1}: \mathbb{T} \rightarrow \mathbb{R}^{n}$. We have

$$
\left\|v_{1}(t)\right\| \leq\left\|x_{1}^{\Delta}(t)\right\|+\rho\left(t, x_{1}(t), x_{1}^{\Delta}(t)\right)
$$

for each $t \in \mathbb{T}$. It follows from Lemma 13 that

$$
\begin{aligned}
\rho\left(t, x_{1}(t), x_{1}^{\Delta}(t)\right) & \leq \rho\left(t, x(t), x^{\Delta}(t)\right)+k(t)\left\|x_{1}(t)-x(t)\right\|+\left\|x_{1}^{\Delta}(t)-x^{\Delta}(t)\right\| \\
& \leq \rho\left(t, x(t), x^{\Delta}(t)\right)+k(t) \rho_{F}(x)+\left\|x_{1}^{\Delta}(t)-x^{\Delta}(t)\right\|
\end{aligned}
$$

and then $v_{1} \in L_{1}\left([a, b)_{\mathbb{T}}, \mathbb{R}^{n}\right)$.

By defining the $\operatorname{arc} x_{2}: \mathbb{T} \rightarrow \mathbb{R}^{n}$ as

$$
x_{2}(t)=x_{0}+\int_{[a, t)_{\mathbb{T}}} v_{1}(\tau) \Delta \tau
$$

we obtain

$$
x_{2}^{\Delta}(t)=v_{1}(t) \in F\left(t, x_{1}(t)\right) \quad \Delta-\text { a.e. } t \in[a, b)_{\mathbb{T}} .
$$

We also have

$$
\begin{aligned}
\left\|x_{2}^{\Delta}(t)-x_{1}^{\Delta}(t)\right\| & =\left\|v_{1}(t)-x_{1}^{\Delta}(t)\right\|=\rho\left(t, x_{1}(t), x_{1}^{\Delta}(t)\right) \\
& \leq \rho\left(t, x(t), x_{1}^{\Delta}(t)\right)+k(t)\left\|x_{1}(t)-x(t)\right\| \\
& \leq k(t) \rho_{F}(x) \quad \Delta-\text { a.e. } \quad t \in[a, b)_{\mathbb{T}}
\end{aligned}
$$

since

$$
x_{1}^{\Delta}(t)=v_{0}(t) \in F(t, x(t)) \quad \Delta-\text { a.e. } t \in[a, b)_{\mathbb{T}} .
$$

Furthermore

$$
\begin{aligned}
\left\|x_{2}(t)-x_{1}(t)\right\| & =\left\|\int_{[a, t)_{\mathbb{T}}}\left(x_{2}^{\Delta}(s)-x_{1}^{\Delta}(s)\right) \Delta s\right\| \\
& \leq \int_{[a, t)_{\mathbb{T}}}\left\|x_{2}^{\Delta}(s)-x_{1}^{\Delta}(s)\right\| \Delta s \leq \rho_{F}(x) \int_{[a, t)_{\mathbb{T}}} k(s) \Delta s
\end{aligned}
$$

for each $t \in \mathbb{T}$.

Continuing the sequence $x_{j}$ as previously, we get at each step

$$
x_{j+1}^{\Delta}(t) \in F\left(t, x_{j}(t)\right) \quad \Delta \text {-a.e. } t \in[a, b)_{\mathbb{T}} .
$$

and

$$
\left\|x_{j+1}^{\Delta}(t)-x_{j}^{\Delta}(t)\right\| \leq k(t)\left\|x_{j}(t)-x_{j-1}(t)\right\| \quad \Delta \text { - a.e. } t \in[a, b)_{\mathbb{T}} .
$$

By mathematical induction we find that

$$
\left\|x_{j+1}^{\Delta}(t)-x_{j}^{\Delta}(t)\right\| \leq \rho_{F}(x) k(t) \alpha(t) \quad \Delta \text { - a.e. } t \in[a, b)_{\mathbb{T}}
$$

and

$$
\left\|x_{j+1}(t)-x_{j}(t)\right\| \leq \rho_{F}(x) \beta(t) \quad \forall t \in \mathbb{T}
$$


where

$$
\alpha(t)=\int_{[a, t)_{\mathbb{T}}} k\left(s_{1}\right) \int_{\left[a, s_{1}\right)_{\mathbb{T}}} k\left(s_{2}\right) \cdots \int_{\left[a, s_{j-2}\right)_{\mathbb{T}}} k\left(s_{j-1}\right) \Delta s_{j-1} \cdots \Delta s_{2} \Delta s_{1}
$$

and

$$
\beta(t)=\int_{[a, t)_{\mathbb{T}}} k\left(s_{1}\right) \int_{\left[a, s_{1}\right)_{\mathbb{T}}} k\left(s_{2}\right) \cdots \int_{\left[a, s_{j-1}\right)_{\mathbb{T}}} k\left(s_{j}\right) \Delta s_{j} \cdots \Delta s_{2} \Delta s_{1} .
$$

From (2) we get

$$
\begin{aligned}
\left\|x_{j+1}^{\Delta}-x_{j}^{\Delta}\right\|_{1} & =\int_{[a, b)_{\mathbb{T}}}\left\|x_{j+1}^{\Delta}(s)-x_{j}^{\Delta}(s)\right\| \Delta s \\
& \leq \int_{[a, b)_{\mathbb{T}}} \rho_{F}(x) k(s) \alpha(s) \Delta s \leq \rho_{F}(x) \frac{\left[\int_{[a, b)_{\mathbb{T}}} k(s) \Delta s\right]^{j}}{j !}
\end{aligned}
$$

and then $\left\{x_{j}^{\Delta}\right\}$ is a Cauchy sequence in $L_{1}\left([a, b)_{\mathbb{T}}, \mathbb{R}^{n}\right)$. Thence there exists $v \in L_{1}\left([a, b)_{\mathbb{T}}, \mathbb{R}^{n}\right)$ satisfying

$$
\left\|x_{j}^{\Delta}-v\right\|_{1}=\int_{[a, b)_{\mathbb{T}}}\left\|x_{j}^{\Delta}(s)-v(s)\right\| \Delta s \rightarrow 0 .
$$

If the arc $y: \mathbb{T} \rightarrow \mathbb{R}^{n}$ is defined by

$$
y(t)=x_{0}+\int_{[a, t)_{\mathrm{T}}} v(s) \Delta s
$$

it follows that

$$
\left\|x_{j}(t)-y(t)\right\|=\left\|\int_{[a, t)_{\mathbb{T}}}\left(x_{j}^{\Delta}(s)-v(s)\right) \Delta s\right\| \leq\left\|x_{j}^{\Delta}-v\right\|_{1}
$$

for all $t \in \mathbb{T}$. Thus, $\left\{x_{j}\right\}$ converges uniformly to the arc $y$.

From Theorem 5 there exists a subsequence $\left\{x_{j_{m}}\right\} \subset\left\{x_{j}\right\}$ such that

$$
x_{j_{m}}^{\Delta}(t) \rightarrow v(t) \quad \Delta-\text { a.e. } t \in[a, b)_{\mathbb{T}} .
$$

Consider $t \in[a, b)_{\mathbb{T}}$ obeying

$$
t \in\left\{s \in[a, b)_{\mathbb{T}}: x_{j+1}^{\Delta}(s) \in F\left(s, x_{j}(s)\right), \forall j\right\}
$$

and

$$
x_{j_{m}}^{\Delta}(t) \rightarrow v(t) .
$$

For each $m \geq 1$ there exist $b_{m} \in B$ and $c_{m} \in F(t, y(t))$ such that

$$
x_{j_{m}}^{\Delta}(t)=c_{m}+\left\|x_{j_{m}-1}(t)-y(t)\right\| b_{m}
$$

and so

$$
v(t)=\lim _{m \rightarrow \infty} x_{j_{m}}^{\Delta}(t)=\lim _{m \rightarrow \infty} c_{m} \in F(t, y(t))
$$

since $F$ is closed.

As

$$
y^{\Delta}(t)=v(t) \quad \Delta-\text { a.e. } \quad t \in[a, b)_{\mathbb{T}}
$$


Revista Ciências Exatas e Naturais, Vol.19, nº.1, Jan/Jun, 2017

we may conclude that

$$
y^{\Delta}(t) \in F(t, y(t)) \quad \Delta-\text { a.e. } t \in[a, b)_{\mathbb{T}} .
$$

Consider $j \geq 2$ arbitrarily fixed. Hence

$$
\begin{aligned}
\left\|x^{\Delta}(s)-y^{\Delta}(s)\right\| & \leq\left\|x^{\Delta}(s)-x_{1}^{\Delta}(s)\right\|+\left\|x_{j+1}^{\Delta}(s)-y^{\Delta}(s)\right\| \\
& +\sum_{l=1}^{j}\left\|x_{l}^{\Delta}(s)-x_{l+1}^{\Delta}(s)\right\| .
\end{aligned}
$$

For each $l \in\{1, \ldots, j\}$ we have

$$
\left\|x_{l+1}^{\Delta}-x_{l}^{\Delta}\right\|_{1} \leq \rho_{F}(x) \frac{\left[\int_{[a, b)_{\mathbb{T}}} k(s) \Delta s\right]^{l}}{l !}
$$

and then

$$
\begin{aligned}
\left\|x^{\Delta}-y^{\Delta}\right\|_{1} & \leq\left\|x^{\Delta}-x_{1}^{\Delta}\right\|_{1}+\left\|x_{j+1}^{\Delta}-y^{\Delta}\right\|_{1} \\
& +\sum_{l=1}^{j}\left\|x_{l}^{\Delta}-x_{l+1}^{\Delta}\right\|_{1} \\
& \leq\left\|x_{j+1}^{\Delta}-y^{\Delta}\right\|_{1}+\rho_{F}(x) \sum_{l=0}^{j} \frac{\left[\int_{[a, b)_{\mathbb{T}}} k(\tau) \Delta \tau\right]^{l}}{l !} \\
& \leq\left\|x_{j+1}^{\Delta}-y^{\Delta}\right\|_{1}+\rho_{F}(x) \exp \left\{\int_{[a, b)_{\mathbb{T}}} k(\tau) \Delta \tau\right\} .
\end{aligned}
$$

Since

$$
\lim _{j \rightarrow \infty}\left\|x_{j+1}^{\Delta}-y^{\Delta}\right\|_{1}=\lim _{j \rightarrow \infty}\left\|x_{j+1}^{\Delta}-v\right\|_{1}=0
$$

it follows that

$$
\|x-y\|_{\infty} \leq\left\|x^{\Delta}-y^{\Delta}\right\|_{1} \leq \rho_{F}(x) \exp \left\{\int_{[a, b)_{\mathbb{T}}} k(\tau) \Delta \tau\right\} .
$$

\section{Conclusion}

This work contributes to the theory of time scales. More specifically, the Theorem 7 provides the existence and approximation of solutions for dynamic inclusions in time scales. Thus, we obtain a generalization of the result [[18], 3.1.6 Theorem]. In the spirit of the present study, [[19], Theorem 1] can be cited as the first result of existence and approximation of solutions to differential inclusions.

\section{References}

[1] ATICI, F., and BILES, D. First order dynamic inclusions on time scales. J Math Anal Appl n.292, v.1, p.222-237. 2004 
[2] BELARBI, A., BENCHOHRA, M., and OUAHAB, A. Existence results for impulsive dynamic inclusions on time scales. Electron J Qual Theory Difer Equ, n.12, p.1-22. 2005

[3] BOHNER, M., and TISDELL, C. Second order dynamic inclusions. J Nonlinear Math Phys v.12, n.2, p.36-45. 2005

[4] CHANG, Y., and LI, W. Existence results for dynamic inclusions on time scales with nonlocal initial conditions. Comput Math Appl v.53, n.1, p.12-20. 2007

[5] FRIGON, M., and GILBERT, H. Systems of rst order inclusions on time scales. Topol Methods Nonlinear Anal v.37, n.1, p.147-163. 2011

[6] GIREJKO, E., and TORRES, D. The existence of solutions for dynamic inclusions on time scales via duality. Appl Math Lett v.25, n.11, p.1632-1637. 2012

[7] SANTOS, I., and SILVA, G. Absolute continuity and existence of solutions to dynamic inclusions in time scales. Math Ann v.356, n.1, p.373-399. 2013

[8] CABADA, A., and VIVERO, D. Expression of the lebesgue $\delta$-integral on time scales as a usual lebesgue integral: application to the calculus of $\delta$-antiderivatives. Math Comput Modelling v.43, n.1-2, 194-207. 2006

[9] BOHNER, M., and PETERSON, A. Dynamic equations on time scales. An introduction with applications. Birkhauser Boston Inc., Boston, MA, 2001.

[10] GUSEINOV, G. Integration on time scales. J Math Anal Appl v.285, n.1, p.107-127. 2003

[11] ROYDEN, H. Real analysis. The Macmillan Co., New York, 1963.

[12] BREZIS, H. Analyse fonctionnelle. Theorie et applications. Masson, Paris, 1983.

[13] , A., and VIVERO, D. Criterions for absolute continuity on time scales. J Diference Equ Appl v.11, n.11, p.1013-1028. 2005

[14] RUDIN, W. Real and complex analysis, 3rd edition. McGraw-Hill Book Co., New York, 1987.

[15] CASTAING, C., and VALADIER, M. Convex analysis and measurable multifunctions. Lecture Notes in Mathematics, vol. 580. Springer-Verlag, Berlin-New York, 1977.

[16] LOEWEN, P. Optimal control via nonsmooth analysis. CRM Proceedings \& Lecture Notes, vol. 2. American Mathematical Society, Providence, RI, 1993.

[17] SANTOS, I., and SILVA, G. Filippov's selection theorem and the existence of solutions for optimal control problems in time scales. Comput Appl Math v.33, n.1, p.223-241. 2014

[18] CLARKE, F. Optimization and nonsmooth analysis. Classics in Applied Mathematics, vol. 5, 2nd edition. Society for Industrial and Applied Mathematics (SIAM), Philadelphia, PA, 1990. 
Revista Ciências Exatas e Naturais, Vol.19, nº.1, Jan/Jun, 2017

[19] FILIPPOV, A. Classical solutions of diferential equations with multi-valued right-hand side. SIAM J Control 5 p.609-621. 1967 